Check for updates

Cite this: RSC Adv., 2019, 9, 13016

Received 25th January 2019

Accepted 21st April 2019

DOI: $10.1039 / c 9 r a 00655 a$

rsc.li/rsc-advances

\title{
Synthesis and characterization of photocrosslinkable hydrogels from bovine skin gelatin
}

\author{
Sanika Suvarnapathaki, ${ }^{\text {ab }}$ Michelle A. Nguyen, (D) ${ }^{\text {bc }}$ Xinchen $\mathrm{Wu}^{\text {ab }}$ \\ Syam P. Nukavarapu iD ${ }^{d}$ and Gulden Camci-Unal (iD *b
}

\begin{abstract}
Hydrogels that mimic native tissues chemically and structurally have been increasingly sought for a wide variety of tissue engineering applications. Gelatin can be naturally derived from different sources and functionalized to fabricate hydrogels that exhibit high cytocompatibility and favorable biodegradable properties. The amino groups on the gelatin backbone can be substituted by adding varying proportions of methacrylic anhydride (MAA) to create biomimetic hydrogels which can be used as tissue engineering scaffolds. Gelatin from different sources yields hydrogels with distinctive physical, chemical, and biological properties. In this work, gelatin from bovine skin was used to fabricate hydrogels with varying degrees of crosslinking content using 1, 4, 7, and $10 \mathrm{~mL}$ MAA. The material properties of these hydrogels were characterized. The cytocompatibility of the gelatin-based hydrogels was studied using L6 rat myoblasts. The hydrogels from bovine skin gelatin exhibit mechanical properties that are conducive for applications which require substrates to propagate cell growth, migration, and proliferation rapidly. These hydrogels exhibit exceptional tunability behavior which makes them useful and applicable to culture different cell types.
\end{abstract}

\section{Introduction}

Fabrication of viable and functional tissue constructs rely on using scaffolding materials that can biologically mimic the native environment. ${ }^{1-7}$ Hydrogels are promising biomaterials because of their ability to mimic the physical, chemical, and biological properties of tissues. ${ }^{8-10}$ Hydrogels that contain biologically active moieties such as collagen, gelatin, and elastin support cell growth, migration, and proliferation. ${ }^{\mathbf{1 1 - 1 4}}$ For example, biological functional groups from proteins, peptides, and growth factors have been shown to provide beneficial effects for cellular interactions as well as improving cell behavior and function. ${ }^{\mathbf{1 5 , 1 6}}$ These biologically active components also allow for chemical interactions with the tissues in vivo including adhesion and integration into the host. ${ }^{17,18}$

Hydrogels from protein-based resources are especially useful due to their functional groups that support cell adhesion. ${ }^{\mathbf{1 9 2 0}}$ Among various protein-based hydrogels, gelatin is a widely used

${ }^{a}$ Biomedical Engineering and Biotechnology Program, University of Massachusetts Lowell, One University Avenue, Lowell, MA, 01854, USA

${ }^{b}$ Department of Chemical Engineering, University of Massachusetts Lowell, One University Avenue, Lowell, MA, 01854,USA.E-mail: Gulden_CamciUnal@uml.edu

'Department of Biomedical Engineering, University of Massachusetts Lowell, One University Avenue, Lowell, MA, 01854, USA

${ }^{d}$ Department of Biomedical Engineering, University of Connecticut, 260 Glenbrook Road, Storrs, CT, 06269, USA naturally-derived material. Gelatin is hydrolyzed from collagen, which is typically, the most abundant protein in the extracellular matrix (ECM) in most tissues. Gelatin degrades enzymatically because of its matrix metalloproteinase (MMP) sensitive protein sequences. Biodegradability over time is a desirable property in tissue engineering applications which require the biomaterial implant to degrade over time for deposition of newly formed ECM by the cells. ${ }^{21-24}$ Moreover, degradation properties of hydrogel scaffolds strongly influence cellular behaviors such as cell spreading, migration, and differentiation. Gelatin is a bioactive material and has been crosslinked using different strategies to form three-dimensional (3D) hydrogels. ${ }^{25}$

Although gelatin can be crosslinked chemically or enzymatically, these methods may require non-physiological conditions and are not suitable for 3D cell encapsulation. ${ }^{26}$ Therefore, practical methods to obtain gelatin-based hydrogels are highly sought after, especially to modulate the pore size, swelling, degradation, and mechanical properties of the scaffold. The chemical modification of gelatin can be performed to synthesize a photocrosslinkable polymer upon exposure to a light source in the presence of a photoinitiator. ${ }^{27}$ Gelatin-based hydrogels have been fabricated using different light sources including ultraviolet (UV) light or visible light (VIS) to form 3D scaffolds. ${ }^{28-32}$ Through varying polymer properties such as the degree of methacrylation, and the physical properties such as the swelling, degradation, compressive modulus, stiffness, and 
tensile strength of gelatin-based hydrogels are easily modulated. ${ }^{33,34}$ Unlike other crosslinking methodologies, photoinitiated crosslinking provides exceptional temporal and spatial control in fabrication of 3D hydrogel scaffolds. This control enables formation of intricate and complex tissue scaffold structures via UV or VIS crosslinking. ${ }^{35}$

Several natural sources can be used to extract gelatin from skins or hides, bones, tendons, and cartilages of different organisms including porcine, fish, and bovine. ${ }^{36,37}$ Porcine skin was the first source used to commercially manufacture gelatin in the 1930 's. ${ }^{38}$ Fish gelatin is primarily derived from their skin and bones. The chemical properties of the fish gelatin differ depending on the specific type of fish. ${ }^{37}$ By using gelatin from different sources, it is possible to obtain hydrogels with a variety of distinct and diverse physical, chemical, and biological properties. This diversity allows the hydrogels to be tailored to a broader range of applications. For instance, bloom strength (also known as gel strength or rigidity), is a critical factor that deviates significantly between porcine and bovine gelatin. The strength of bovine skin gelatin (BSG) and porcine skin gelatin (PSG) increases proportionally with $\mathrm{pH}$. However, in PSG, the gel strength increases inconsistently with $\mathrm{pH}^{39}$ With less rigidity, improved cell spreading is expected within the hydrogels made from BSG. ${ }^{39}$

In this work, we synthesized photocrosslinkable hydrogels from bovine skin gelatin and extensively characterized their swelling, degradation, porosity, mechanical properties, and biological response in vitro.

\section{Materials and methods}

\section{Materials}

Dulbecco's phosphate buffered saline (DPBS), Dulbecco's modified Eagle's medium (DMEM-low glucose), fetal bovine serum (FBS), trypsin-ethylenediaminetetraacetic acid, and penicillin/streptomycin were purchased from Gibco (Thermo Fisher Scientific, Inc., Waltham, MA). Alamar Blue reagent was obtained from Invitrogen (Grand Island, NY). 2-Hydroxy-1-[4(hydroxyethoxy)phenyl]-2-methyl-1-propanone (Irgacure 2959) was purchased from BASF Corporation (Florham Park, NJ). Methacrylic anhydride was purchased from Sigma-Aldrich (St. Louis, MO). Bovine skin-derived gelatin and Dulbecco's Phosphate Buffer Saline (PBS) modified without calcium chloride and magnesium chloride were purchased from Sigma-Aldrich (St. Louis, MO). All reagents were used as received without further purification.

\section{Synthesis of BGelMA}

The hydrogel precursor was composed of $5 \%(\mathrm{w} / \mathrm{v})$ bovine GelMA (BGelMA) and 0.5\% (w/v) Irgacure 2959 (photoinitiator). Four different degrees of chemical modification via a methacrylation reaction for BGelMA were achieved by using $1 \mathrm{~mL}, 4$ $\mathrm{mL}, 7 \mathrm{~mL}$, and $10 \mathrm{~mL}(\mathrm{v} / \mathrm{v})$ MAA. BGelMA was synthesized by combining $10 \mathrm{~g}$ of bovine skin gelatin with $100 \mathrm{~mL}$ of Phosphate Buffered Saline (PBS) at $50{ }^{\circ} \mathrm{C}$. Four batches of this mixture were stirred continuously until the gelatin was completely dissolved in the PBS. To achieve different degrees of methacrylation, 1, 4, 7 , and $10 \mathrm{~mL}$ of methacrylic anhydride were added dropwise to the corresponding bovine gelatin mixture. The gelatin mixtures were in reaction for 4 hours under constant stirring at $200 \mathrm{rpm}$. Each mixture was then diluted with $300 \mathrm{~mL}$ of PBS to stop the methacrylation reaction. The mixtures were dialyzed against distilled water for one week under stirring $(180 \mathrm{rpm})$ at $40{ }^{\circ} \mathrm{C}$. After dialysis, these solutions were frozen overnight at $-80{ }^{\circ} \mathrm{C}$ and subsequently lyophilized for a week to obtain the BGelMA. During the freeze-drying process, the end product formed a foam, which was used to make the prepolymer solutions. The degrees of methacrylation in each polymer condition were quantified through proton $\left({ }^{1} \mathrm{H}\right)$ NMR analysis.

\section{Swelling and degradation analysis}

For swelling analysis, the samples were prepared by pipetting $100 \mu \mathrm{L}$ of $5 \%(\mathrm{w} / \mathrm{v})$ prepolymer in $0.5 \%(\mathrm{w} / \mathrm{v})$ photoinitiator solution. The solutions were crosslinked under UV light using an Omnicure S2000 (EXFO Photonic Solutions Inc., Ontario, Canada). The exposure times for UV crosslinking were optimized for each condition of MAA. The gels that were modified with 1, 4, 7, and $10 \mathrm{~mL}$ MAA were crosslinked at 92, 77, 60, and 37 seconds, respectively. The crosslinked hydrogels were then submerged in Petri dishes containing either PBS, deionized water, or Dulbecco's Modified Eagle Medium (DMEM) for 48 hours to reach equilibrium swelling. Four replicates for each MAA condition were tested in each liquid (i.e. PBS, deionized water, or DMEM). After reaching swelling equilibrium, the wet weight of each hydrogel was measured after gently removing the excess liquid using Kimwipes. Then, each hydrogel was placed in an Eppendorf tube, weighed, frozen, and lyophilized for 24 hours. The dry weights of the hydrogels were recorded after lyophilization. Swelling ratio was calculated by dividing the wet weights of the gels by their corresponding dry weights; the resulting number was converted into a percentage value.

For degradation analysis, the samples were fabricated using the same method described for the swelling analysis. The empty Eppendorf tubes were weighed prior to transferring the gels into them. The gels in the Eppendorf tubes were frozen overnight and subsequently lyophilized for 24 hours. Then, the dry weight of the gels was recorded by subtracting the weight of the empty Eppendorf tube from the total weight of the dried gel in the Eppendorf tube. The lyophilized gels were rehydrated in $1 \mathrm{~mL}$ of PBS for 24 hours. Four replicates were fabricated for each hydrogel composition. The PBS was removed from the Eppendorf tube and $1 \mathrm{~mL}$ of $2.5 \mathrm{U} \mathrm{mL}^{-1}$ of collagenase type II solution in PBS was added to the gels. The samples were incubated at $37{ }^{\circ} \mathrm{C}$ on a shaker at $70 \mathrm{rpm}$. The amount of degradation was measured at different time points (i.e. 3, 6, 12, 18, 24, 36, and 48 hours). The samples were washed with PBS three times to ensure that the enzyme solution was completely removed at each time point. Subsequently, the gels were frozen overnight before lyophilization. Dry weight of the gels was recorded after degradation. The percent mass remaining after degradation was quantified by dividing the dry weight after degradation with 
the initial weight of the hydrogel. The resulting values were then converted and reported as a percentage value.

\section{Mechanical testing}

In mechanical analysis, the BGelMA hydrogel samples were prepared using the same process as described in the previous sections on swelling and degradation analysis such that there were four replicates per MAA condition. After fabrication of the crosslinked hydrogels, the samples were allowed to swell in PBS for 24 hours. Before performing the compression test, the samples were punched using an $8 \mathrm{~mm}$ biopsy punch. The excess liquid on the gels was removed gently by using Kimwipes. The compression test was conducted by applying preload force of $0.0010 \mathrm{~N}$ at an isothermal temperature of $23{ }^{\circ} \mathrm{C}$, soak time of 1 minute, and force ramp rate of $0.1 \mathrm{~N} \mathrm{~min}^{-1}$. The upper force limit was set to $2 \mathrm{~N}$. The compressive modulus of each sample was determined by obtaining the slope in the linear region of the stress-strain curve.

\section{Pore size determination}

The hydrogel samples were analyzed for their pore size using scanning electron microscopy (SEM). Specifically, a JEOL 5200 SEM was used for morphological characterization of the BGelMA samples. The gel samples were flash frozen in liquid nitrogen, freeze dried, and coated with gold under an argon atmosphere. SEM images were analyzed using the NIH Image J for determining the percent porosity and pore size for gels with $1,4,7$, and $10 \mathrm{~mL}$ MAA concentrations.

\section{Three-dimensional (3D) cell encapsulation in BGelMA hydrogels}

For cytocompatibility studies, L6 rat myoblast cells were encapsulated in the hydrogel precursor solution using a cell seeding density of $5 \times 10^{6}$ cells per $\mathrm{mL}$. The hydrogel precursor solutions were prepared with $5 \%(\mathrm{w} / \mathrm{v})$ for each hydrogel composition that were synthesized by the addition of $1,4,7$, and, $10 \mathrm{~mL}$ of MAA. To prepare for 3D encapsulation of the $\mathrm{L} 6$ rat myoblasts, the cells were trypsinised from the flask, transferred into a conical tube, and centrifuged to form a pellet. The cell count was obtained from the cell pellet to determine appropriate amounts of cells for homogenous resuspension in the different prepolymer solutions. Then, $10 \mu \mathrm{L}$ of prepolymer solution containing cells was pipetted on a Petri dish and covered with a glass side. The hydrogels were photocrosslinked using the UV light at $2.5 \mathrm{~W} \mathrm{~cm}^{-2}$ power (Fig. 1). The microencapsulated cell-laden gels were then rinsed with PBS and cultured in standard 24 culture plates for a period of seven days. The samples were then analyzed on days $0,1,4$, and 7 .

\section{Cell viability and morphology}

The biocompatibility of the BGelMA hydrogels with different quantities of MAA was assessed by studying the viability and metabolic activity of L6 rat myoblasts. The L6 cells were cultured in the DMEM medium supplemented with $10 \%(\mathrm{v} / \mathrm{v})$ fetal bovine serum (FBS) and 5\% (v/v) penicillin/streptomycin.

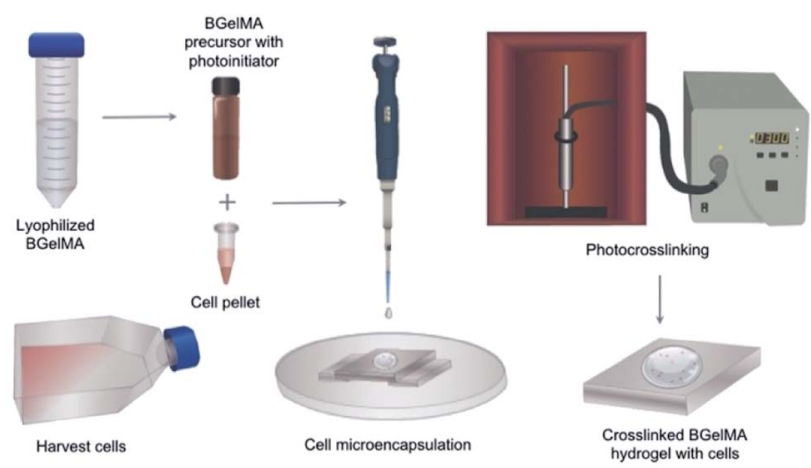

Fig. 1 Schematic for the cell microencapsulation protocol. The L6 rat myoblast cells were trypsinized and centrifuged to obtain a cell pellet. The cells were resuspended in 5\% (w/v) BGelMA. The cell-laden prepolymer solution was then UV crosslinked.

The L6 rat myoblast cultures were maintained in a $37{ }^{\circ} \mathrm{C}$ incubator with $5 \%$ carbon dioxide $\left(\mathrm{CO}_{2}\right)$. The media was changed every $2-3$ days.

The viability of the cells was determined by using a live/dead assay which contained calcein AM and propidium iodide. The viability test was performed according to the manufacturer's protocol. $^{40,41}$ The number of live and the number of dead cells were quantified at each time point (day 1, 4, and 7) by image analysis using the ImageJ software.

The metabolic activity of the cells was measured by performing an Alamar Blue assay. ${ }^{42}$ The Alamar blue solution was incubated with the cells for 4 hours. The colorimetric results were read using a microplate reader in the fluorescence detection mode. The fluorescence values of the resulting solutions were recorded at $560 \mathrm{~nm} / 590 \mathrm{~nm}(\mathrm{Ex} / \mathrm{Em})$. Cell morphology and spreading were studied by staining the samples with Texas Red Phalloidin and DAPI (4',6-diamidino-2-phenylindole). The images showed the cell morphology at different time points (days 1, 4, and 7) for three replicates of each 1, 4, 7, and $10 \mathrm{~mL}$ MAA-modified BGelMA samples.

\section{Statistical analysis}

All statistical analyses were performed using the GraphPad Prism v 6.0 (La Jolla, CA, USA). One-way ANOVA analyses was carried out with Bonferroni tests. The data sets were presented as average \pm standard deviation $\left({ }^{*} p<0.05,{ }^{* *} p<0.01,{ }^{* * *} p<\right.$ $0.001, * * * * p<0.0001)$.

\section{Results and discussion}

The physical properties of the photocrosslinkable gelatin-based hydrogels vary depending on the degree of methacrylate substitution, polymer concentration $(\mathrm{w} / \mathrm{v})$, photoinitiator concentration $(\mathrm{w} / \mathrm{v})$, and the light exposure time. The photoinitiator concentration and the UV exposure time are critical parameters that must be optimized to ensure cytocompatibility ranges. ${ }^{27}$

The BGelMA with four different degrees of methacrylation were synthesized using $1,4,7$, and $10 \mathrm{~mL}$ MAA per $10 \mathrm{~g}$ of 
bovine gelatin for each condition. Fig. 2 shows the ${ }^{1} \mathrm{H}$ NMR spectrum for a BGelMA sample with $10 \mathrm{~mL}$ MAA. The peak integration values for the protons of the methacrylate vinyl groups (double bonds) of methacrylic anhydride and the protons of the aromatic amino acid residues of BGelMA are provided on the figure. The degree of methacrylation is defined as the ratio of the amino groups that are functionalized with methacrylate groups to the total number of amino groups in bovine gelatin before the methacrylation reaction. ${ }^{43,44}$ To determine the degree of methacrylation, integration value under the peaks for the double bond region is compared with the integration value under the peaks in the aromatic region. Therefore, the degree of methacrylation was calculated by dividing the area under the peaks $\delta=5.4-5.8 \mathrm{ppm}$ by the area under the peak $\delta=7.4 \mathrm{ppm}$ as described previously. ${ }^{43,44}$ Using the ${ }^{1} \mathrm{H}$ NMR spectrum in Fig. 2, the following calculation was performed for the samples with $10 \mathrm{~mL}$ MAA: $(0.44+0.44) / 1 \times$ $100 \%$ which yielded an $88 \%$ degree of methacrylation. The degree of methacrylation for each condition was determined as $35 \%, 69 \%, 79 \%$, and $88 \%$, respectively using the ${ }^{1} \mathrm{H}$ NMR data. The BGelMA hydrogels that were fabricated using these methacrylated precursors yielded scaffolds tailored for tissue engineering applications requiring cell spreading and migration within the matrix. The physical properties of these hydrogels were studied as well as the viability and metabolic activity behaviors of the cells encapsulated within these hydrogels. The results of the different biocompatibility analyses show that BGelMA hydrogel is a functionally viable and effective scaffolding material in tissue engineering applications.

\section{Swelling analysis for BGelMA hydrogels}

The hydrogel network is a $3 \mathrm{D}$ crosslinked matrix, allowing it to hold water in its microstructure and swell. This dynamic ability is due to the hydrophilic nature of the hydrogels. The degree to which the hydrogels swell can have a direct impact on the behavior of cells encapsulated within the scaffold; thus, swelling properties are important to characterize.

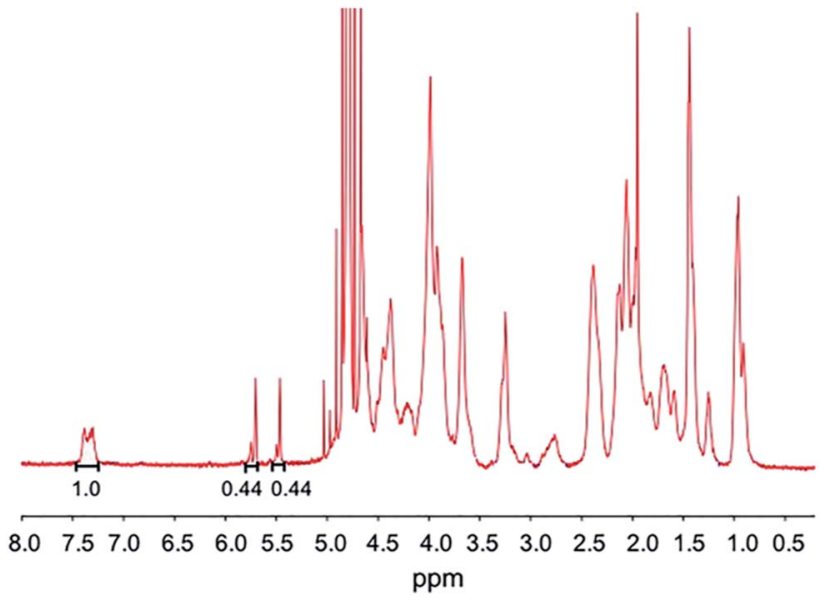

Fig. $2{ }^{1} \mathrm{H}$ NMR spectrum of BGelMA with $10 \mathrm{~mL}$ MAA.
In this study, the swelling behavior of the BGelMA hydrogels was characterized in deionized water, PBS, and DMEM media. The swelling ratios of these hydrogels showed a consistently increasing trend in swelling in all of the liquids tested as the degree of methacrylation decreased (Fig. 3A and B). The hydrogels swelled the most in deionized water and the least in cell growth media for all of the gel compositions. The BGelMA

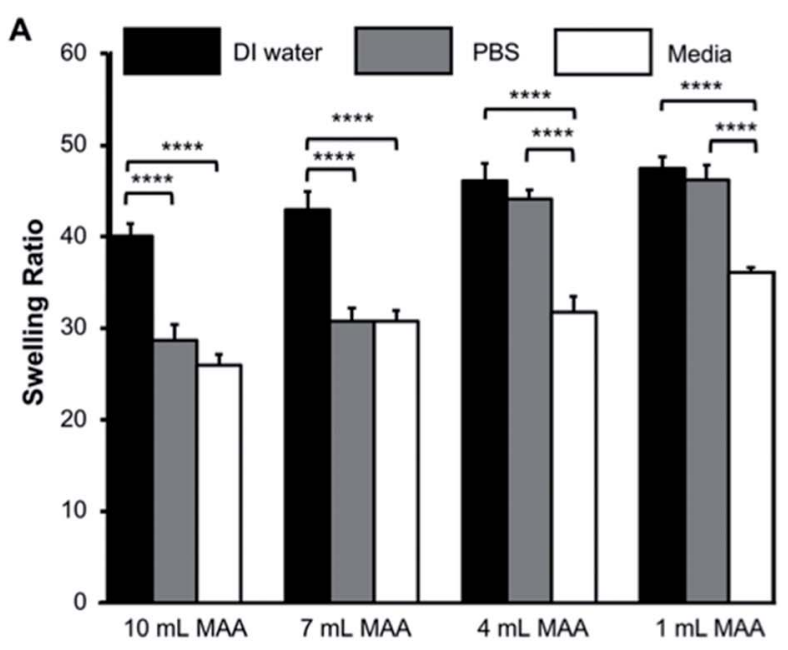

B
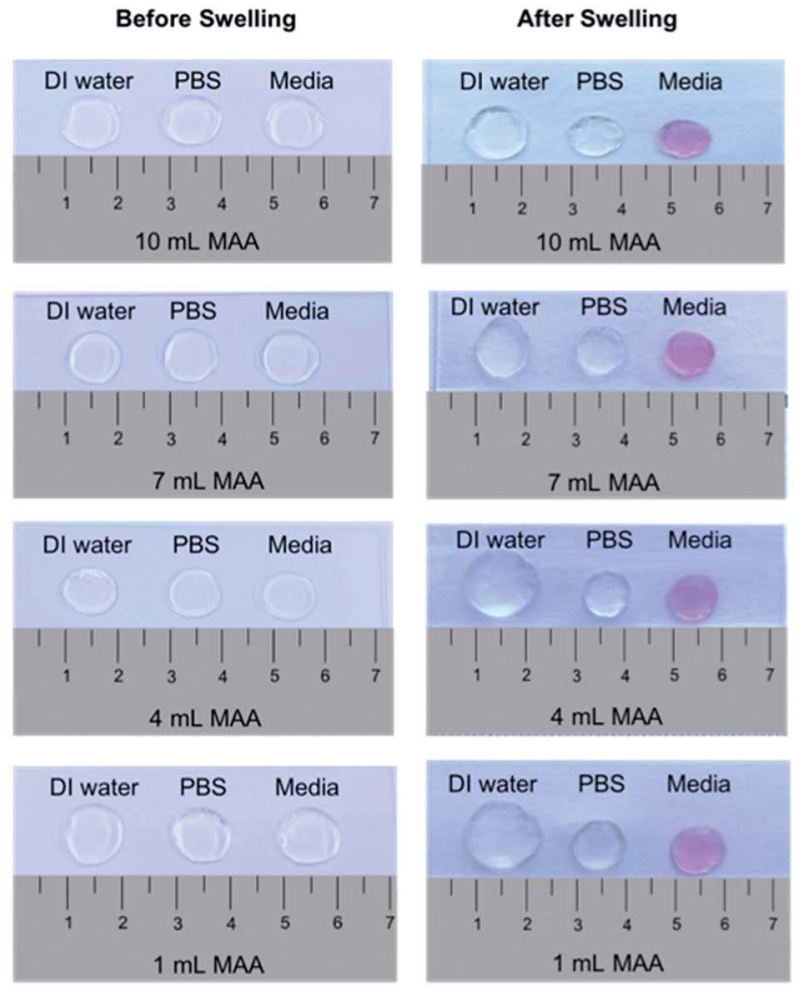

Fig. 3 Swelling properties of bovine skin gelatin-based hydrogels with varying degrees of methacrylation. (A) The swelling ratios of the hydrogel scaffolds with $10,7,4$, and $1 \mathrm{~mL}$ MAA per $10 \mathrm{~g}$ of gelatin in deionized water, PBS, and DMEM. (B) Images of the gels before and after reaching to equilibrium swelling in deionized water, PBS, and DMEM. 
hydrogels with the highest degree of methacrylation $(10 \mathrm{~mL}$ MAA) was highly crosslinked and had the smallest pores, therefore, they swelled the least. In contrast, the hydrogels with the lowest degree of methacrylation ( $1 \mathrm{~mL}$ MAA) were loosely crosslinked and had the largest pores which caused the highest swelling. The swelling ratios for the hydrogels with $10 \mathrm{~mL}$ MAA were $40.0 \pm 1.3$ in deionized water, $28.6 \pm 1.7$ in PBS, and $25.8 \pm$ 1.1 in DMEM. For the gels with $7 \mathrm{~mL}$ MAA, the swelling ratios were $42.9 \pm 1.8$ in deionized water, $30.7 \pm 1.4$ in PBS, and $30.7 \pm$ 1.1 in DMEM. The hydrogels that were modified using $4 \mathrm{~mL}$ MAA had a swelling profile of $46.0 \pm 3.1$ in deionized water, 44.1 \pm 1.4 in PBS, and $31.7 \pm 1.5$ in DMEM. In the gels with $1 \mathrm{~mL}$ MAA, swelling ratios were $47.4 \pm 1.2$ in deionized water, $46.2 \pm$ 1.6 in PBS, and $36.0 \pm 0.5$ in DMEM (Fig. 3A). Our results demonstrated that swelling behavior was significantly impacted by the solution in which the samples were incubated to swell. We attribute this behavior to the physical properties of the hydrogel matrix. Properties such as permeability and diffusion coefficient affect how certain liquids to enter the matrix. ${ }^{45}$ In addition, the tested liquids have different viscosities that would impact how much liquid volume the hydrogel networks can occupy. Deionized water is the least viscous of the solutions tested; therefore, it can diffuse more readily into the hydrogel matrix. PBS has a higher salt concentration and is relatively more viscous than water. As a result, the hydrogels swelled less in PBS than in deionized water. The DMEM media includes salts and proteins and is thereby the most viscous one among the tested liquids. As expected, the hydrogels swelled the least in the DMEM media.

Our results demonstrated that the BGelMA hydrogels offer a wide range of tunability in their swelling characteristics (Fig. 3A). As hypothesized, the swelling ratios calculated for the BGelMA with $1 \mathrm{~mL}$ MAA were significantly higher than that of the gels with $4 \mathrm{~mL}, 7 \mathrm{~mL}$, and $10 \mathrm{~mL}$ MAA modification in all of the liquids tested. We attribute this behavior to the chemical and physical properties of the hydrogel matrix. Swelling behavior increases or decreases depending on the degree of substitution of the amino groups on the gelatin backbone. Varied degrees of substitution are achieved by adding different ratios of methacrylic anhydride to bovine gelatin during the methacrylation reaction. The substitution affects the ability of the polymer chains to form crosslinks. The gel samples with lower quantities of methacrylic anhydride have lower degrees of substitution. Therefore, fewer crosslinks are formed in the hydrogel which results in weaker mechanical properties. The swelling ratios of the gels are higher due to their ability to retain more water within their matrix. Gel samples with higher amounts of methacrylic anhydride form more crosslinks, resulting in stronger mechanical properties as reported in the previous studies. ${ }^{4-49}$ In these gels, the higher number of crosslinks provide less space to retain water as they swell and therefore result in lower swelling ratios. The polymer crosslinking density also can be controlled by adjusting the UVexposure time, concentration of the photoinitiator, and the concentration of the BGelMA prepolymer. The degree of substitution was examined for each condition using ${ }^{1} \mathrm{H}$ NMR. Fig. 2 shows a representative ${ }^{1} \mathrm{H}$ NMR spectrum for a sample with $10 \mathrm{~mL}$ MAA. According to the integration of the peaks, the degree of substitution was calculated to be $88 \%$. The impact of degree of substitution on the swelling behavior is shown quantitatively by the ${ }^{1} \mathrm{H}$ NMR and swelling analyses.

\section{Degradation analysis for BGelMA hydrogels}

Gelatin is composed of matrix metalloproteinase (MMP)sensitive degradation sites which allow for gradual degradation under physiological conditions. Hydrogel degradation over time is critical because it enables the cells that are encapsulated in the hydrogel to deposit their ECM within the 3D scaffold. In the body, degradation of hydrogels is influenced by several factors such as enzymes, water sensitive groups, $\mathrm{pH}$, and other biological factors. For example, collagenase is an enzyme found in the body that degrades collagen. Since gelatin is a form of hydrolyzed collagen, it degrades hydrogels made from a gelatin backbone.

In this work, the degradation behavior was assessed for all hydrogel compositions of BGelMA. Enzymatic degradation was performed using $2.5 \mathrm{U} \mathrm{mL}^{-1}$ collagenase type II at $37^{\circ} \mathrm{C}$ during a 48 hour period. The degraded mass was calculated at 3, 6, 12, 18, 24, 36, and 48 hours (Fig. 4). Results in Fig. 4 showed that the $\%$ mass that remained for hydrogels that were modified using $10 \mathrm{~mL}$ of MAA included 87\%, 79\%, 69\%, 66\%, 59\%, 56\%, and $49 \%$ after $3,6,12,18,24,36$, and 48 hours of degradation, respectively. The \% gel mass in these hydrogels decreased gradually as time elapsed, as expected. In the BGelMA samples with $7 \mathrm{~mL}$ of MAA, $79 \%, 71 \%, 65 \%, 61 \%, 56 \%, 52 \%$, and $37 \%$ of the gel mass remained at $3,6,12,18,24,36$, and 48 hours, respectively. Similarly, the \% mass that remained at each time point continuously decreased in the collagenase solution. These hydrogels also did not fully degrade within the 48 hour period. For the hydrogels that contained $4 \mathrm{~mL}$ of MAA, at 3, 6, 12, 18, 24, 36 , and 48 hours, $69 \%, 62 \%, 51 \%, 41 \%, 36 \%, 20 \%$, and $7 \%$ of gel mass remained, respectively. Again, the results showed a consistent trend of gradual decrease in the \% gel mass in the

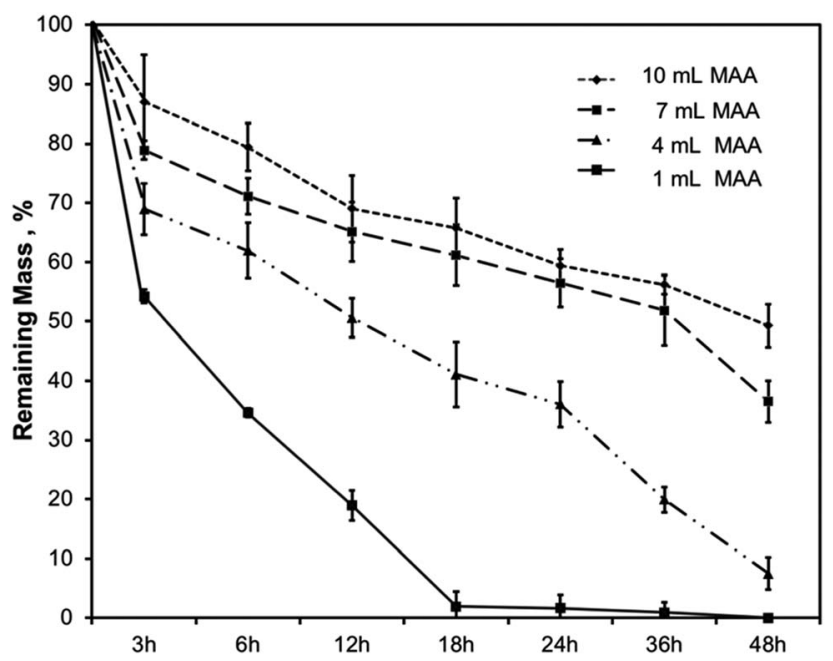

Fig. 4 Degradation characteristics of the BGelMA with 10, 7, 4, and $1 \mathrm{~mL}$ MAA content in collagenase type II. 
48 hour period without reaching full degradation. In the hydrogels with $1 \mathrm{~mL}$ MAA, 54\%, 35\%, 19\%, 2\%, 1\%, and $0 \%$ of gel mass remained at $3,6,12,18,24,36$, and 48 hours. In contrast to the previous conditions, the hydrogels fully degraded in a 48 hour period at a more rapid rate compared to the scaffolds with different amounts of MAA. The mechanically weakest gels from the BGelMA with $1 \mathrm{~mL}$ MAA tended to degrade most rapidly while the mechanically most robust gels that were modified with $10 \mathrm{~mL}$ MAA degraded later in the enzymatic degradation solution. In the gels with higher amounts of MAA, there is a higher degree of methacrylation which provides larger number of crosslinking sites. These hydrogels can form more robust polymer crosslinks forming mechanically stronger end products that allow the gel to withstand degradation for longer time. Based on the higher MAA content, the gels can understandably degrade at a much slower rate than the mechanically weaker gels with lower amounts of MAA.

\section{Mechanical characteristics of BGelMA hydrogels}

The compressive moduli of the BGelMA hydrogels were determined using a dynamic mechanical analyzer. The mechanical properties of a hydrogel are contingent on the gel's crosslinking density which considerably influences cell spreading within the hydrogel network. Specifically, the mechanics of the gel strongly influences cell morphology, cell-cell interactions, and cell spreading within the 3D scaffold.

The compressive moduli increased with the increase in the amount of MAA in the BGelMA hydrogels (Fig. 5). The trend observed in our experiments is consistent with previously reported studies. ${ }^{49,50}$ The compressive moduli were $0.7 \mathrm{kPa} \pm 0.1$, $0.8 \mathrm{kPa} \pm 0.2,1.4 \mathrm{kPa} \pm 1.0$, and $2.3 \mathrm{kPa} \pm 1.0$ for the hydrogels with $1,4,7$, and $10 \mathrm{~mL}$ MAA, respectively. The mechanical strength of the hydrogel matrix is dependent on the degree of methacrylation, the concentration of the prepolymer solution, and the UV crosslinking time. In our study, the degree of methacrylation was varied by changing the amount of MAA (1,

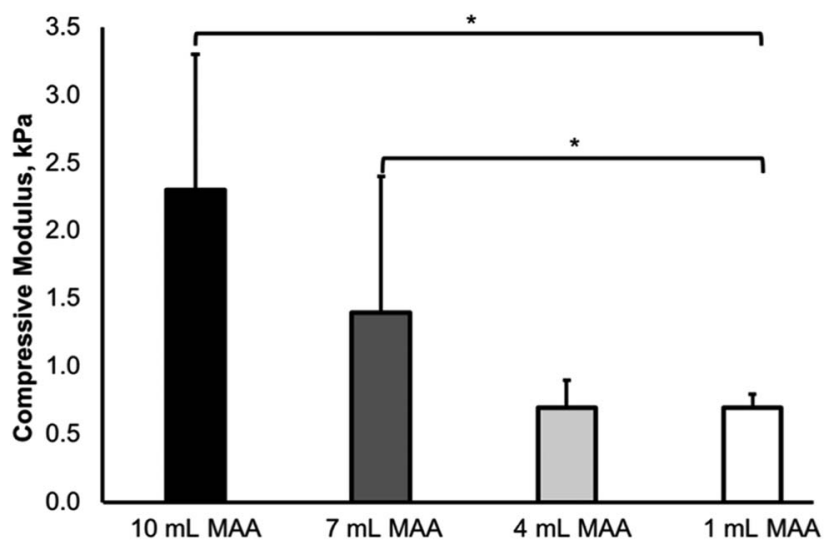

Fig. 5 Mechanical characterization of the BGelMA hydrogels at different concentrations of MAA. The compressive moduli of the BGelMA hydrogels were found to be highly tunable. The data sets are presented as average \pm standard deviation $(* p<0.05)$.
4,7 , and $10 \mathrm{~mL}$ per $10 \mathrm{~g}$ of bovine gelatin). With higher degrees of methacrylation, increasing numbers of polymer crosslinks are formed, yielding a mechanically stronger gel. The compressive moduli of these hydrogels were in the expected range compared with retrospective studies that tested gelatinbased hydrogels. ${ }^{21}$

\section{SEM analysis and evaluation of pore size}

The SEM images of the BGelMA samples were acquired at $5 \mathrm{kV}$ after freeze-drying and sputter-coating them with gold. The pore size and percent porosity of the BGelMA samples with different degrees of methacrylation were determined using the NIH ImageJ (Fig. 6). The \% porosity decreased with increasing amounts of MAA, as expected. Higher methacrylation results in the \% porosity values which were calculated as $80.9 \pm 2.6,63.9$ $\pm 5.1,48.6 \pm 2.8$, and $30.0 \pm 1.2$, for the gels with $1,4,7$, and $10 \mathrm{~mL}$ MAA respectively (Fig. 6E). As expected, \% porosity decreased as the methacrylation increased due to the increase in crosslinking density.

In addition to \% porosity, average pore sizes were also determined for photocrosslinkable BGelMA hydrogels. The average pore sizes for the samples were $12.6 \mu \mathrm{m} \pm 4.6(1 \mathrm{~mL}$ MAA), $7.3 \mu \mathrm{m}$ \pm 2.7 (4 mL MAA), $5.2 \mu \mathrm{m} \pm 1.5$ (7 mL MAA), and $3.6 \mu \mathrm{m} \pm 1.1$ (10 mL MAA) (Fig. 6F). A one-way ANOVA was performed for the data which showed significant differences between each degree of methacrylation. Because the gels with higher degree of methacrylation generate higher numbers of crosslinks, they form denser matrices in the final products. The pore sizes are relatively small in highly dense hydrogels. Conversely, in the gels with lower degrees of methacrylation, fewer crosslinks are generated and less dense hydrogel matrices are formed which result in relatively large pore sizes. This phenomenon is evidently shown in the SEM images in Fig. 6A-D.

\section{Cell response to BGelMA hydrogels in 3D}

The physical properties such as the swelling, degradation, porosity, mechanical properties, and the degree of chemical modification significantly impact the cellular behavior within the hydrogel scaffolds in 3D. We have shown that the BGelMA hydrogels with different degrees of methacrylation exhibited distinguishable effects on cellular viability, metabolic activity, migration, and cell morphology.

The viability of the L6 rat myoblasts that were encapsulated in the BGelMA hydrogels was determined using the images from fluorescently stained cell-laden microgels using a live/ dead assay (Fig. 7). Green fluorescent staining represents the live cells that were stained with calcein AM and red fluorescent staining shows the dead cells that were stained with propidium iodide. Fluorescent images were captured using an inverted microscope and the images were analyzed using the NIH ImageJ. Three replicates were quantified in ImageJ per condition at each time point. The percent viabilities were calculated using the following formula:

$$
\begin{aligned}
\% \text { viability }= & {[(\text { live cell intensity }) /(\text { live cell intensity }} \\
& + \text { dead cell intensity })] \times 100
\end{aligned}
$$



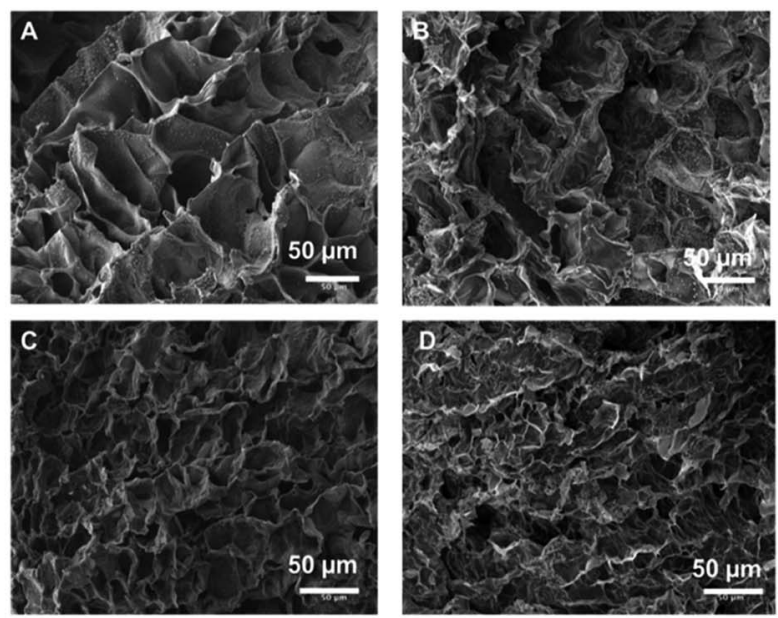

E
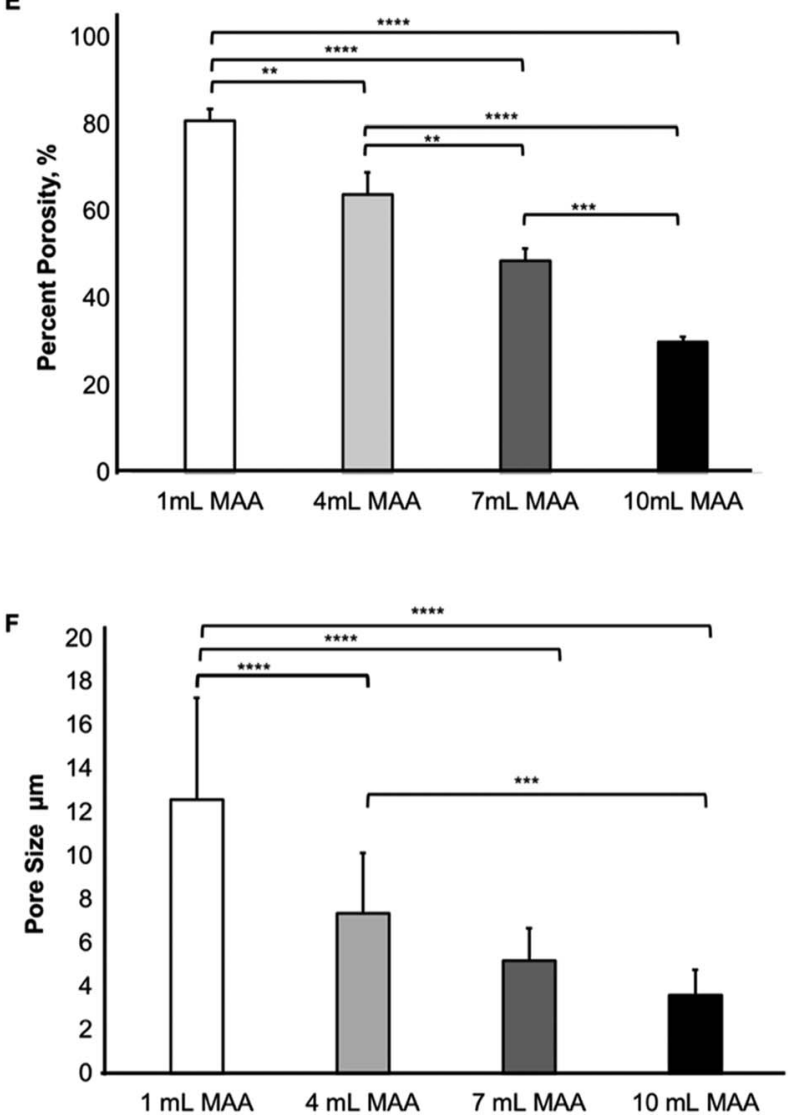

Fig. 6 Scanning Electron Microscopy (SEM) analysis for 5\% (w/v) BGelMA hydrogels with (A) $1 \mathrm{~mL}$, (B) $4 \mathrm{~mL}$, (C) $7 \mathrm{~mL}$ and (D) $10 \mathrm{~mL} M A A$, and $(E)$ percent porosity in hydrogels with different concentrations of MAA. (F) Average pore size for the gels with different amounts of MAA The data sets are presented as averages \pm standard deviation $(* * * p<$ $0.001, * * * * p<0.0001)$

The viability values were then averaged and reported for days 1, 4, and 7. The percent viability of cells for the BGelMA hydrogels with $10 \mathrm{~mL}$ MAA were $93.5 \pm 3.7,85.4 \pm 8.2$, and 80.8 \pm 5.1 for days 1,4 , and 7 , respectively. For the samples with $7 \mathrm{~mL}$ MAA, the percent cell viability was found to be $95.2 \pm 1.7$, $86.7 \pm 8.8$, and $80.6 \pm 2.5$ on days 1,4 , and 7 , respectively. The
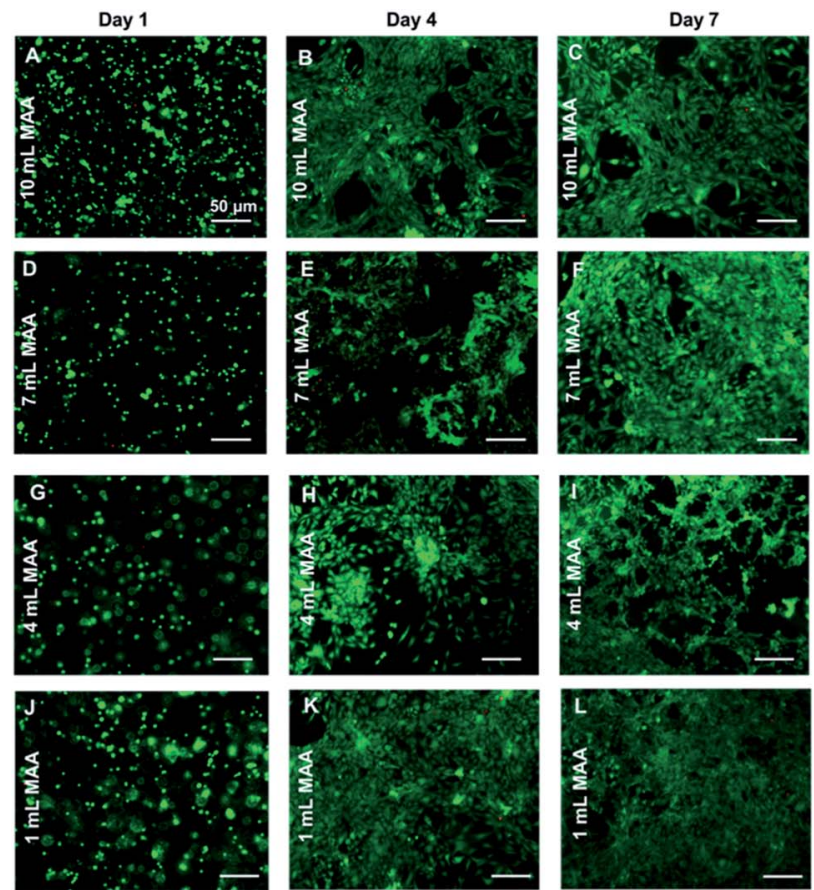

M
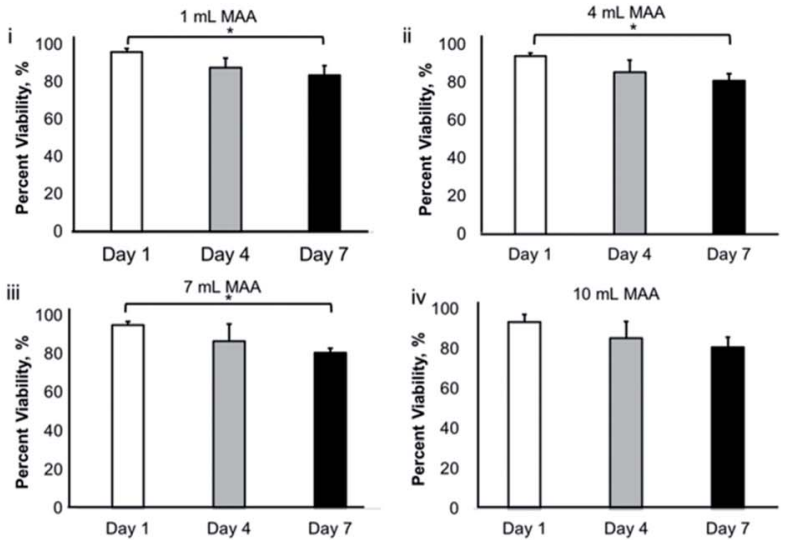

Fig. 7 Viability of L6 rat myoblasts encapsulated in 5\% (w/v) BGelMA hydrogels with different quantities of MAA. Fluorescent images were acquired for the samples that were stained using calcein AM (green) and propidium iodide (red). The fluorescent images were captured on days 1,4 , and 7 for the gels with $10 \mathrm{~mL}$ MAA (A-C), $7 \mathrm{~mL}$ MAA (D-F), $4 \mathrm{~mL} M A A(G-I)$, and $1 \mathrm{~mL} M A A(J-L)$. (M (i-iv)) Percent viability of the L6 rat myoblasts in BGelMA gels. Image analyses were performed using $\mathrm{NIH}$ ImageJ for calculation of \% viability. Scale bar: $50 \mu \mathrm{m}$.

hydrogels that were modified using $4 \mathrm{~mL}$ MAA yielded in percent viabilities of $94.1 \pm 1.5,85.5 \pm 6.5$, and $80.9 \pm 3.8$, for days 1,4 , and 7, respectively. Lastly, the percent viability values for the hydrogels with $1 \mathrm{~mL}$ MAA were $96.0 \pm 1.9,87.8 \pm 4.9$, and $83.6 \pm 5.0$, for day 1,4 , and 7 , respectively. The viability results showed that a high cell viability was maintained over the one-week culture period. The viability was significantly higher on day 1 (over 94\%) compared to day 7 (over 81\%). Overall, high cell viabilities were obtained in bovine gelatin-based hydrogels in each degree of methacrylation. The positive cell response indicates the suitability of these matrices as 3D scaffolds for cell cultures. 
In addition to cell viability, we also stained for F-actin (Texas Red Phalloidin) and nuclei (DAPI) of the cells to visualize their cytoskeletal arrangement in the BGelMA hydrogels in 3D. The L6 rat myoblasts demonstrated round shapes in every condition on day 1 (Fig. 8). No cell spreading was seen on day 1 as expected due to the early time point. The cells were found to progressively spread over time in all four gel compositions. The L6 cells spread the most in the gels that have $1 \mathrm{~mL}$ MAA compared to the gels with 4 mL MAA. The amount of spreading decreased as the amount of MAA increased. This result can be attributed to MAA content, pore size, and mechanical properties of the hydrogel matrix. The gels with lower amounts of MAA forms fewer crosslinks and hence softer gels. The softer gel with larger pores provides the cells more room in 3D to spread. This behavior was supported by our results. The fluorescent images revealed that the L6 rat myoblasts were highly spread within all gel compositions by day 7 . The gels with lower ratios of MAA form fewer crosslinks and hence softer gels.

The metabolic activity of the L6 rat myoblasts in BGelMA hydrogels with different degrees of methacrylation was tested using the Alamar Blue assay. Fig. 9 illustrates an increase in the metabolic activity for each condition over 7 days of culture period. The lowest metabolic activity was observed on day 1 which then steadily increased on day 4 . The highest metabolic activity was then found on day 7 . This trend was seen for all hydrogel conditions that contained 1, 4, 7, and, 10 mL MAA. However, the metabolic activity in the gels with $10 \mathrm{~mL}$ MAA was determined to be significantly higher in comparison to the metabolic activity of the rest of the conditions.

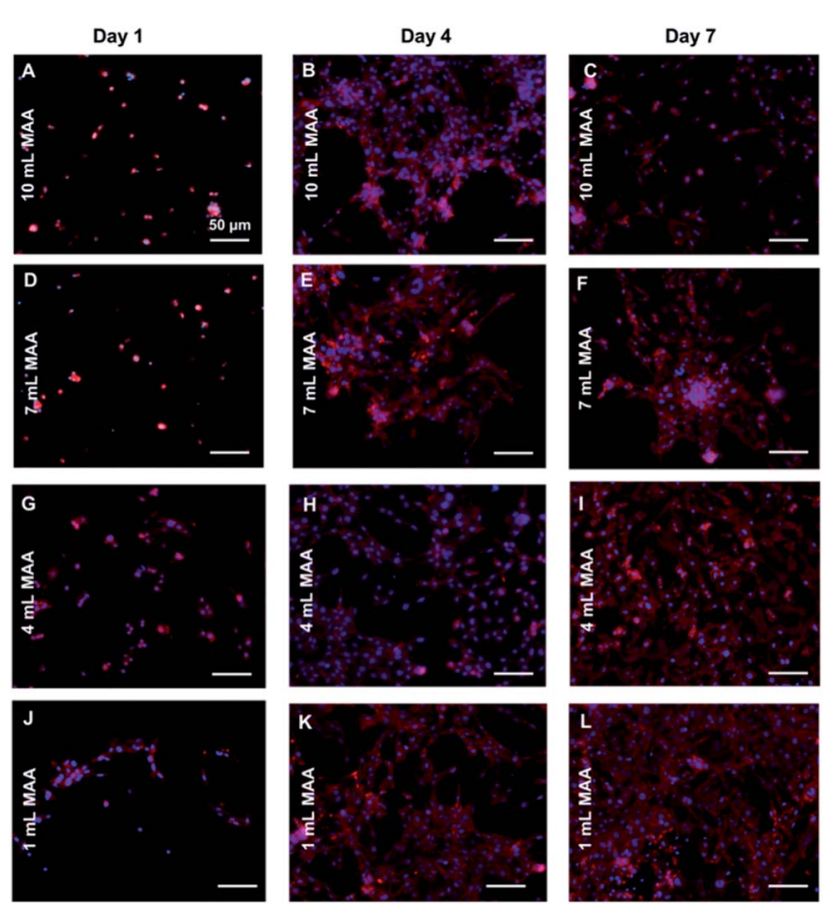

Fig. 8 Cytoskeleton and nuclei staining with Phalloidin and DAPI for the $L 6$ rat myoblast cells that were 3D encapsulated within $5 \%(\mathrm{w} / \mathrm{v})$ BGelMA hydrogels. Fluorescent images were captured on days 1, 4, and 7 for the gels that contained $10 \mathrm{~mL}$ MAA (A-C), $7 \mathrm{~mL}$ MAA (D-F), $4 \mathrm{~mL}$ MAA $(\mathrm{G}-\mathrm{I})$, and $1 \mathrm{~mL}$ MAA $(\mathrm{J}-\mathrm{L})$. Scale bar: $50 \mu \mathrm{m}$.

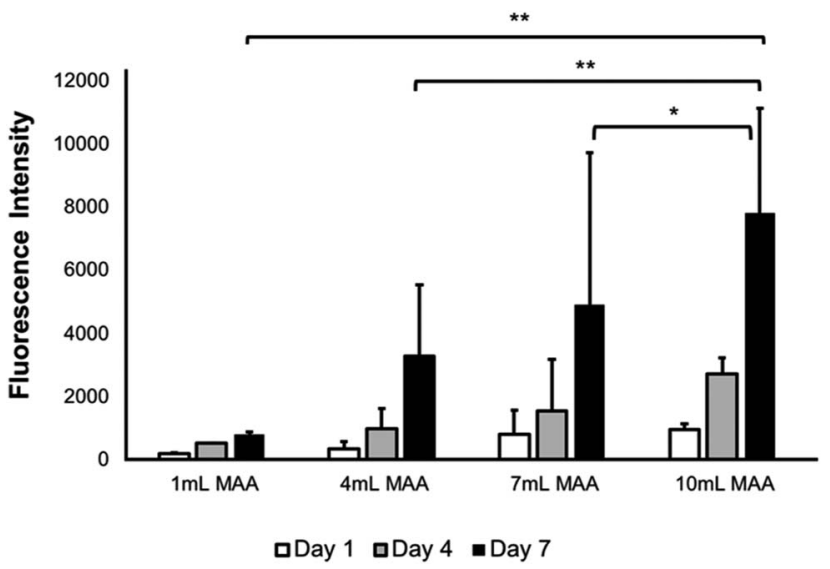

Fig. 9 Proliferation of L6 rat myoblasts within BGelMA hydrogels with different degrees of methacrylation studied using an Alamar Blue assay. A progressive increase in proliferation was found in all BGelMA conditions with different amounts of MAA. Error bars: $\pm \mathrm{SD},(* p<0.05$, $* * p<0.01)$.

\section{Conclusions}

Gelatin is highly accessible from multiple natural sources and can be easily chemically modified to fabricate bioactive scaffolds. ${ }^{51,52}$ This work discusses the synthesis and characterization of hydrogels made from methacrylation of gelatin from bovine skin (BGelMA). These hydrogels were successfully used as 3D scaffolds to support the growth, viability, migration, and metabolic response of L6 rat myoblast cells in BGelMA with different degrees of methacrylation. We extensively characterized the physical (swelling, degradation, compressive strength, pore size, porosity), and biological properties (cell viability, growth, migration, metabolic activity) of these hydrogels. The compressive modulus did significantly impact the behavior of cells encapsulated within them. In mechanical tests, the stiffer gels with higher amounts of MAA possessed higher compressive modulus than gels that were less stiff and were composed of lower volumes of MAA. The use of gelatin from a source other than the standard porcine skin gelatin yielded different mechanical and biological properties while maintaining high flexibility and tunability. Such high tunability is a desirable feature for various tissue engineering applications and fabrication of clinically translatable tissue products. The unique mechanical properties of the BGelMA hydrogels allow improved cell spreading, migration, and interactions. These qualities found in the BGelMA hydrogels satisfy a vast range of parameters required in the fabrication of scaffolds for engineering soft tissue constructs.

\section{Conflicts of interest}

There are no conflicts to declare.

\section{Acknowledgements}

This research was supported by the UMass Lowell Faculty StartUp Funds. We acknowledge Dr Wendy Gavin, Ms Julia N. Renaghan, and Mr Akhil Meka for technical help. 


\section{References}

1 J. M. Karp, P. D. Dalton and M. S. Shoichet, Scaffolds for tissue engineering, MRS Bull., 2003, 28(4), 301-306.

2 G. Camci-Unal, A. Laromaine, E. Hong, R. Derda and G. M. Whitesides, Biomineralization guided by paper templates, Sci. Rep., 2016, 6, 27693.

3 M. J. Hancock, F. Piraino, G. Camci-Unal, M. Rasponi and A. Khademhosseini, Anisotropic material synthesis by capillary flow in a fluid stripe, Biomaterials, 2011, 32(27), 6493-6504.

4 D. Lantigua, Y. N. Kelly, B. Unal and G. Camci-Unal, Engineered paper-based cell culture platforms, $A d v$. Healthcare Mater., 2017, 6(22), 1700619.

5 X. Wu, S. Suvarnapathaki, K. Walsh and G. Camci-Unal, Paper as a scaffold for cell cultures: Teaching an old material new tricks, MRS Commun., 2018, 8(1), 1-14.

6 G. Camci-Unal, J. W. Nichol, H. Bae, H. Tekin, J. Bischoff and A. Khademhosseini, Hydrogel surfaces to promote attachment and spreading of endothelial progenitor cells, J. Tissue Eng. Regener. Med., 2013, 7(5), 337-347.

7 G. Camci-Unal, N. Annabi, M. R. Dokmeci, P. Liao and A. Khademhosseini, Hydrogels for cardiac tissue engineering, NPG Asia Mater., 2014, 6(5), e99.

8 K. Y. Lee and D. J. Mooney, Hydrogels for tissue engineering, Chem. Rev., 2001, 101(7), 1869-1880.

9 M. Bauer, L. Kang, Y. Qiu, J. Wu, M. P. Howard, H. Chen and G. Camci-Unal, Adult cardiac progenitor cell aggregates exhibit survival benefit both in vitro and in vivo, PLoS One, 2012, 7(11), e50491.

10 G. Camci-Unal and A. Khademhosseini, Biomaterials that breathe, Chem. Ind., 2013, 77(8), 28-30.

11 W. E. Hennink and C. F. van Nostrum, Novel crosslinking methods to design hydrogels, Adv. Drug Delivery Rev., 2012, 64, 223-236.

12 A. N. Wilson and A. Guiseppi-Elie, Bioresponsive hydrogels, Adv. Healthcare Mater., 2013, 2(4), 520-532.

13 J. M. Harris, M. D. Bentley, X. Zhoa and X. Shen, Hydrolytically degradable polymers and hydrogels made therefrom, US Pat., No. 6,348,558, 2002.

14 T. Miyata, T. Uragami and K. Nakamae, Biomoleculesensitive hydrogels, Adv. Drug Delivery Rev., 2002, 54(1), 7998.

15 K. Smetana Jr, Cell biology of hydrogels, Biomaterials, 1993, 14(14), 1046-1050.

$16 \mathrm{Z}$. Wei and S. Gerecht, A self-healing hydrogel as an injectable instructive carrier for cellular morphogenesis, Biomaterials, 2018, 185, 86-96.

17 G. C. Ingavle, M. Gionet-Gonzales, C. E. Vorwald, L. K. Bohannon, K. Clark, L. D. Galuppo and J. K. Leach, Injectable mineralized microsphere-loaded composite hydrogels for bone repair in a sheep bone defect model, Biomaterials, 2019, 197, 119-128.

18 T. T. Lee, J. R. García, J. I. Paez, A. Singh, E. A. Phelps, S. Weis and A. J. García, Light-triggered in vivo activation of adhesive peptides regulates cell adhesion, inflammation and vascularization of biomaterials, Nat. Mater., 2015, 14(3), 352.

19 N. Annabi, J. W. Nichol, X. Zhong, C. Ji, S. Koshy, A. Khademhosseini and F. Dehghani, Controlling the porosity and microarchitecture of hydrogels for tissue engineering, Tissue Eng., Part B, 2010, 16(4), 371-383.

20 K. T. Nguyen and J. L. West, Photopolymerizable hydrogels for tissue engineering applications, Biomaterials, 2002, 23(22), 4307-4314.

21 G. Camci-Unal, D. Cuttica, N. Annabi, D. Demarchi and A. Khademhosseini, Synthesis and characterization of hybrid hyaluronic acid-gelatin hydrogels, Biomacromolecules, 2013, 14(4), 1085-1092.

22 F. Yanagawa, S. Sugiura, T. Takagi, K. Sumaru, G. CamciUnal, A. Patel and T. Kanamori, Activated-Ester-Type Photocleavable Crosslinker for Preparation of Photodegradable Hydrogels Using a Two-Component Mixing Reaction, Adv. Healthcare Mater., 2015, 4(2), 246-254.

23 P. Dubruel, R. Unger, S. V. Vlierberghe, V. Cnudde, P. Jacobs, J. E. Schacht and C. J. Kirkpatrick, Porous gelatin hydrogels: 2. In vitro cell interaction study, Biomacromolecules, 2007, 8(2), 338-344.

24 G. D. Nicodemus and S. J. Bryant, Cell encapsulation in biodegradable hydrogels for tissue engineering applications, Tissue Eng., Part B, 2008, 14(2), 149-165.

$25 \mathrm{Y}$. Liu and M. B. Chan-Park, Hydrogel based on interpenetrating polymer networks of dextran and gelatin for vascular tissue engineering, Biomaterials, 2009, 30(2), 196-207.

26 J. Berger, M. Reist, J. M. Mayer, O. Felt, N. A. Peppas and R. Gurny, Structure and interactions in covalently and ionically crosslinked chitosan hydrogels for biomedical applications, Eur. J. Pharm. Biopharm., 2004, 57(1), 19-34.

27 K. Yue, G. T. Santiago, M. M. Alvarez, A. Tamayol, N. Annabi and A. Khademhosseini, Synthesis, properties, and biomedical applications of gelatin methacryloyl (GelMA) hydrogels, Biomaterials, 2015, 73, 254-271.

28 J. Maitra and V. K. Shukla, Cross-linking in hydrogelsa review, Am. J. Polym. Sci., 2014, 4(2), 25-31.

29 R. Bhat and A. Karim, Ultraviolet irradiation improves gel strength of fish gelatin, Food Chem., 2009, 113(4), 1160-1164.

30 W. Xiao, J. He, J. W. Nichol, L. Wang, C. B. Hutson and A. Khademhosseini, Synthesis and characterization of photocrosslinkable gelatin and silk fibroin interpenetrating polymer network hydrogels, Acta Biomater., 2011, 7(6), 2384-2393.

31 L. Hockaday, A. Kang, K. H. Colangelo, N. W. Cheyung and C. C. Chu, Rapid 3D printing of anatomically accurate and mechanically heterogeneous aortic valve hydrogel scaffolds, Biofabrication, 2012, 4(3), 035005.

32 S. T. Koshy, R. M. Desai, P. Joly, J. Li and D. J. Mooney, ClickCrosslinked Injectable Gelatin Hydrogels, Adv. Healthcare Mater., 2016, 5(5), 541-547.

33 B. Hertenstein, A. Bernd, K. C. Wollert, M. Hofmann and H. Drexler, Monitoring of Bone Marrow Cell Homing in the Infarcted Human Myocardium by PET, Blood, 2004, 111(17), 2696. 
34 A. I. Van De Bulcke, B. Bogdanov, D. Rooze and H. Berghmans, Structural and rheological properties of methacrylamide modified gelatin hydrogels, Biomacromolecules, 2000, 1(1), 31-38.

35 B. J. Klotz, D. Gawlitta, A. J. Rosenberg, J. Malda and F. P. Melchels, Gelatin-methacryloyl hydrogels: towards biofabrication-based tissue repair, Trends Biotechnol., 2016, 34(5), 394-407.

36 A. Khademhosseini and R. Langer, Microengineered hydrogels for tissue engineering, Biomaterials, 2007, 28(34), 5087-5092.

37 B. Jamilah and K. Harvinder, Properties of gelatins from skins of fish-black tilapia (Oreochromis mossambicus) and red tilapia (Oreochromis nilotica), Food Chem., 2002, 77(1), 81-84.

38 M. Gómez-Guillén and M. P. Montero, Functional and bioactive properties of collagen and gelatin from alternative sources: A review, Food Hydrocolloids, 2011, 25(8), 1813-1827.

39 R. Hafidz, C. M. Yaakob, I. Amin and A. Noorfaizan, Chemical and functional properties of bovine and porcine skin gelatin, Int. Food Res. J., 2011, 813-817.

40 D. Bratosin, L. Mitrofan, C. Palii and J. Montreuil, Novel fluorescence assay using calcein-AM for the determination of human erythrocyte viability and aging, Cytometry, Part A, 2005, 66(1), 78-84.

41 T. Crompton, M. C. Peistsch, H. R. MacDonald and J. Tschopp, Propidium iodide staining correlates with the extent of DNA degradation in isolated nuclei, Biochem. Biophys. Res. Commun., 1992, 183(2), 532-537.

42 S. Al-Nasiry, N. Geusens, M. Hanssens and R. Pijnenborg, The use of Alamar Blue assay for quantitative analysis of viability, migration and invasion of choriocarcinoma cells, Hum. Reprod., 2007, 22(5), 1304-1309.

$43 \mathrm{H}$. Shin, B. D. Olsen and A. Khademhosseini, The mechanical properties and cytotoxicity of cell-laden double-network hydrogels based on photocrosslinkable gelatin and gellan gum biomacromolecules, Biomaterials, 2012, 33(11), 3143-3152.

44 W. T. Brinkman, K. Nagapudi, B. S. Thomas and E. L. Chaikof, Photo-cross-linking of type I collagen gels in the presence of smooth muscle cells: mechanical properties, cell viability, and function, Biomacromolecules, 2003, 4(4), 890-895.

45 M. F. Refojo, Permeation of water through some hydrogels, $J$. Appl. Polym. Sci., 1965, 9(10), 3417-3426.

46 H. Wang, L. Zhou, J. Liao, Y. Tan, K. Ouyang, C. Ning and G. Tan, Cell-laden photocrosslinked GelMA-DexMA copolymer hydrogels with tunable mechanical properties for tissue engineering, J. Mater. Sci.: Mater. Med., 2014, 25(9), 2173-2183.

47 S. Suvarnapathaki, R. Ramos, S. W. Sawyer, S. McLoughlin, A. Ramos, S. Venn and P. Soman, Generation of cell-laden hydrogel microspheres using 3D printing-enabled microfluidics, J. Mater. Res., 2018, 33(14), 2012-2018.

48 S. A. Bencherif, A. Srinivasan, F. Horkay, J. O. Hollinger, K. Matyjaszewski and N. R. Washburn, Influence of the degree of methacrylation on hyaluronic acid hydrogels properties, Biomaterials, 2008, 29(12), 1739-1749.

49 C. A. Bonino, J. E. Samorezov, O. Jeon, E. Alsberg and S. A. Khan, Real-time in situ rheology of alginate hydrogel photocrosslinking, Soft Matter, 2011, 7(24), 11510-11517.

50 Y. C. Chen, R. Z. Lin, H. Qi, Y. Yang and A. Khademhosseini, Functional human vascular network generated in photocrosslinkable gelatin methacrylate hydrogels, Adv. Funct. Mater., 2012, 22(10), 2027-2039.

51 U. T. Rashid, Gelatin-Based Hydrogels, Cellulose-Based Superabsorbent Hydrogels, Polymers and Polymer Composites: A Reference Series, Springer, Cham, 2018, pp. 1-41.

52 V. Crescenzi, A. Francescangeli and A. Taglienti, New gelatinbased hydrogels via enzymatic networking, Biomacromolecules, 2002, 3(6), 1384-1391. 